\title{
Daratumumab improves the anti-myeloma effect of newly emerging multidrug therapies
}

This article was published in the following Dove Press journal:

Blood and Lymphatic Cancer:Targets and Therapy

17 April 2013

Number of times this article has been viewed

\author{
Sathish Gopalakrishnan' \\ Daryl Tan ${ }^{1,2}$ \\ 'Department of Hematology, \\ Singapore General Hospital, \\ Singapore, Republic of Singapore; \\ ${ }^{2}$ Raffles Cancer Center, Raffles \\ Hospital, Singapore, Republic \\ of Singapore
}

\begin{abstract}
Although the clinical outcomes of patients with multiple myeloma has improved tremendously with the advent of bortezomib and immunomodulatory drugs like thalidomide and lenalidomide, the disease remains incurable and patients will eventually be resistant to these drugs. Novel non-cross-resistant modalities of treatment are needed. Immunotherapy is potentially a very promising therapeutic modality for further development. Daratumumab is a novel, high-affinity, therapeutic human monoclonal antibody against a unique CD38 epitope. It induces tumor-cell killing through several immunological mechanisms. It has shown a favorable safety profile as monotherapy and significant single-agent activity in relapsed/refractory myeloma. It has also demonstrated strong synergism with lenalidomide and bortezomib. The potential of this agent, together with its pharmacokinetics, mode of action, early efficacy, and safety data will be detailed in this review.
\end{abstract}

Keywords: daratumumab, myeloma, monoclonal

\section{Introduction}

\section{Management issues in the treatment of multiple myeloma}

Multiple myeloma (MM) is a plasma cell malignancy, and the second most common hematological neoplasm. Its incidence increases significantly with age with a median age of diagnosis of about 70 years. ${ }^{1}$ Since the 1990 s, we have seen improved clinical outcomes among younger patients with the introduction of autologous stem cell transplantation (ASCT). Of late, the advent of novel agents, including the immunomodulatory drugs (IMiDs) thalidomide and lenalidomide and the proteasome inhibitor bortezomib, has revolutionized the treatment paradigm for MM. Survival outcomes for elderly patients who are not eligible for ASCT have also improved tremendously with novel agents. ${ }^{2-5}$ Currently, IMiDs and bortezomib are increasingly being incorporated into standard first-line regimens for treatment of MM, and they have resulted in improved disease outcomes compared with the disease outcomes of standard upfront regimens. However, most patients will still eventually relapse or become refractory to these agents whether received as part of first-line or second-line therapy. In the era of novel therapy, although MM patients are enjoying longer survivals, we are beginning to see more resistant and atypical relapses. ${ }^{6,7}$ Salvaging such patients becomes particularly challenging and their prognosis often remains dismal.

The treatment of myeloma is evolving rapidly. In addition to the five main active classes of drugs - alkylators (eg, melphalan and cyclophosphamide), corticosteroids (eg, prednisone and dexamethasone), proteasome inhibitors (eg, bortezomib and carfilzomib), IMiDs (eg, thalidomide and lenalidomide), and anthracyclines 
(eg, doxorubicin and liposomal doxorubicin) - that form the backbone of MM therapy, there remains a need for agents with novel modes of action. The addition of a different modality of treatment to the therapeutic armamentarium against MM will give us a better chance at overcoming drug resistance.

\section{Immunotherapy in MM}

A promising treatment route is the development of immunotherapeutic approaches to target and eliminate MM cells more selectively. Allogeneic transplantation, the most established of immunotherapeutic techniques, may result in durable responses due to antitumor immunity mediated by donor lymphocytes. However, the morbidity and mortality related to graft-versus-host disease remain a challenge; this approach cannot be recommended as a standard of care and should only be carried out for carefully selected patients in the context of a clinical study. An important prerequisite for immunotherapy to be effective is the expression of tumorassociated antigens (TAAs) on tumor cells. Vaccination strategies targeting single antigens like the idiotype or whole cells have shown promise in clinical studies. Idiotype-loaded dendritic cells (DCs) have been used by various groups as vaccines to consolidate remissions after ASCT. ${ }^{8-12}$ However, to date, there is still no compelling evidence from these Phase I and II studies that the natural course of MM can be altered by vaccination approaches. It is also unlikely that vaccination approaches can be applied on a wide routine basis. In light of the success of rituximab in the treatment of B-cell non-Hodgkin lymphoma, perhaps a more robust way of harnessing the immunogenicity of TAAs and eliciting myeloma-specific immune responses to eliminate the malignant plasma cell is through the development of therapeutic monoclonal antibodies (mAbs). Ideally, a useful target for mAb-based MM therapy should be expressed exclusively on the majority of the MM cells. A wide range of antigens may be targeted in MM therapy and they include cell surface receptors, signaling molecules involved in cell survival, antiapoptotic pathways and cell-to-cell communication, and the interactions between MM cells and bone marrow stromal cells. ${ }^{13}$ Several diverse mAbs are currently being evaluated in preclinical and clinical studies. Mechanisms of action of these mAbs include antibody-dependent cellular cytotoxicity (ADCC), complement-dependent cytotoxicity (CDC), interference with receptor-ligand interactions, and $\mathrm{mAb}$ conjugation to radioisotopes or toxins. ${ }^{14}$ Promising mAbs now being tested in clinical trials include elotuzumab (anti-CS1), siltuximab (anti-IL-6), and BT062 (anti-CD138). At present, elotuzumab is probably the most advanced in clinical development. Interestingly, as a single agent, its antitumor activity is only modest, but when combined with lenalidomide, an IMiD, overall response rates in excess of $80 \%$ are seen in Phase I and II studies. ${ }^{15-17}$ Elotuzumab is now being investigated in a randomized Phase III trial in combination with lenalidomide and dexamethasone. Daratumumab is another novel $\mathrm{mAb}$ developed for the treatment of MM. Originally developed by Genmab (Copenhagen, Denmark), a global license and development agreement with Janssen Biotech, Inc (Horsham, PA, USA) was announced in September 2012. Daratumumab is a high-affinity, therapeutic human monoclonal antibody against a unique CD38 epitope. The potential of this agent, together with its pharmacokinetics, mode of action, early efficacy, and safety data, will be covered in detail in this review.

\section{CD38 and its potential for targeting}

CD38 is a small multifunctional glycoprotein that is expressed at relatively low levels on lymphoid and myeloid lineages, but is absent from most mature resting lymphocytes. ${ }^{18}$ It is a $46 \mathrm{kDa}$ type II transmembrane glycoprotein with a short 20-aa N-terminal cytoplasmic tail and a long 256-aa extracellular domain. ${ }^{19}$ It serves not only as an antigen but also as an enzyme that catalyzes the metabolism of cyclic adenosine diphosphate ribose and nicotinic acid adenine dinucleotide phosphate, two structurally and functionally distinct calcium messengers that regulate the endoplasmic reticulum and lysosomal calcium stores, respectively. ${ }^{20}$ In most cases of MM, CD38 is highly expressed on the cell surface. Coupled with its role in cell signaling, CD38 has been identified as an attractive target for therapeutic mAbs. ${ }^{21,22}$

Several mAbs to human CD38 that induce killing of neoplastic B cell lines have been generated. ${ }^{23,24}$ Two antiCD38 antibodies are in clinical development at present. One is the humanized monoclonal antibody SAR650984 and the second one is daratumumab, a human anti-CD38 antibody that is generated in transgenic mice (Figure 1).

\section{Review of pharmacology, mode of action, pharmacokinetics, safety, tolerability, and efficacy}

Daratumumab has broad-spectrum killing activity. It is able to induce killing of tumor cells via anti-Fc-mediated effector functions, CDC, natural killer cell-mediated ADCC, antibody-dependent cellular phagocytosis, and apoptosis upon secondary cross-linking (Figure 2). ${ }^{25,26}$ It stands out from other CD38 mAbs in its strong ability to induce CDC in patient 


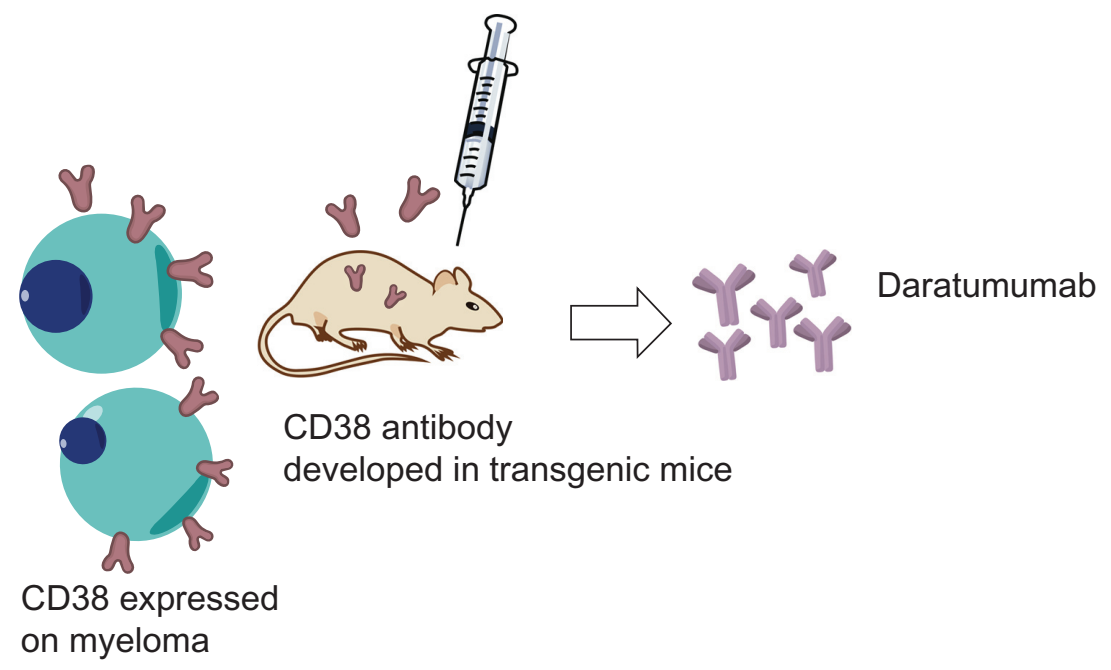

Figure I Depiction of development of daratumumab by immunization of transgenic mice with CD38 molecule.

MM cells. Importantly, daratumumab-induced ADCC and CDC were not affected by the presence of bone marrow stromal cells, suggesting that it can effectively kill MM cells in a tumorpreserving bone marrow microenvironment. ${ }^{25}$ In vivo xenograft studies ${ }^{24,25}$ have also demonstrated that daratumumab is highly active and is able to interrupt tumor growth at low dosing levels. Recent data has also shown that daratumumab is effective against MM cells derived from therapy-naïve or -refractory patients grafted in a humanized mouse model that closely simulates MM with its bone marrow microenvironment in the human body. ${ }^{27}$ As targeting MM cells through various mechanisms is the primary goal of combination strategies aiming to overcome the development of drug resistance, daratumumab was further explored on the bench to demonstrate its potential when combined with other agents. A combination of daratumumab with lenalidomide was found to be highly synergistic. In a series of experiments using purified MM cells, MM cell lines, full bone marrow mononuclear cell suspensions derived from MM patients, and peripheral blood mononuclear cells isolated from lenalidomide-treated patients, the combination was shown to further augment cell killing as compared to the cell killing seen with either of the agents alone. ${ }^{28}$ This is consistent with the observation seen with elotuzumab, whereby the administration of lenalidomide further stimulates the effector cells to enhance antibody-dependent lysis of MM cells. ${ }^{16}$ The impact of combining daratumumab with two of the most promising frontline regimens in MM therapy, namely, lenalidomide/bortezomib with dexamethasone (revlimide/velcade/dexamethasone [RVD] regimen) and bortezomib/melphalan with prednisolone (velcade/melphala/prednisolone [VMP] regimen), has been further explored preclinically. ${ }^{29}$ This involved using serial dilutions of prepared combinations of these agents alone or in the presence of daratumumab and incubating with MM cell lines and bone marrow mononuclear cells of MM patients. Addition of daratumumab was shown to almost double the cell killing potential of these two regimens. ${ }^{29}$

There are now abundant preclinical data that show the versatility of daratumumab in killing CD38-expressing tumor cells effectively via diverse cytotoxic mechanisms and provide the proof of concept for the development of the drug as a single agent and in combination with other agents in the clinical arena. ${ }^{25,27-29}$

Daratumumab is currently being investigated in a Phase I/II safety- and dose-finding study for the treatment of patients with MM. ${ }^{30-33}$ Inclusion criteria for this study include patients who are $>18$ years and ineligible for ASCT, and have relapsed or are refractory to at least 2 different prior therapies. The study was based on a $3+3$ doseescalation design. Daratumumab was administered over a 9-week period consisting of two pre- and seven full doses, with doses ranging from $0.005 \mathrm{mg} / \mathrm{kg}$ to $24 \mathrm{mg} / \mathrm{kg}$. Steroids were administered to prevent infusion-related events up to a maximum dose equivalent to $27 \mathrm{mg}$ dexamethasone per week. Updated results for 32 patients enrolled so far have been recently reported. ${ }^{33}$

The most common adverse events reported were infusionrelated events (IREs) including bronchospasm, headache, dyspnea, and fever. The observed IREs occurred predominantly during the initial infusions. Nine percent of patients reported IREs during the pre-dose while $26 \%$ had IREs during the first full infusion. A gradual decrease in frequency was seen after the second full infusion. No dose relationship between dose and IRE was observed. Most IREs occurred within 3-4 hours of infusion. Two patients had grade 3 IREs 


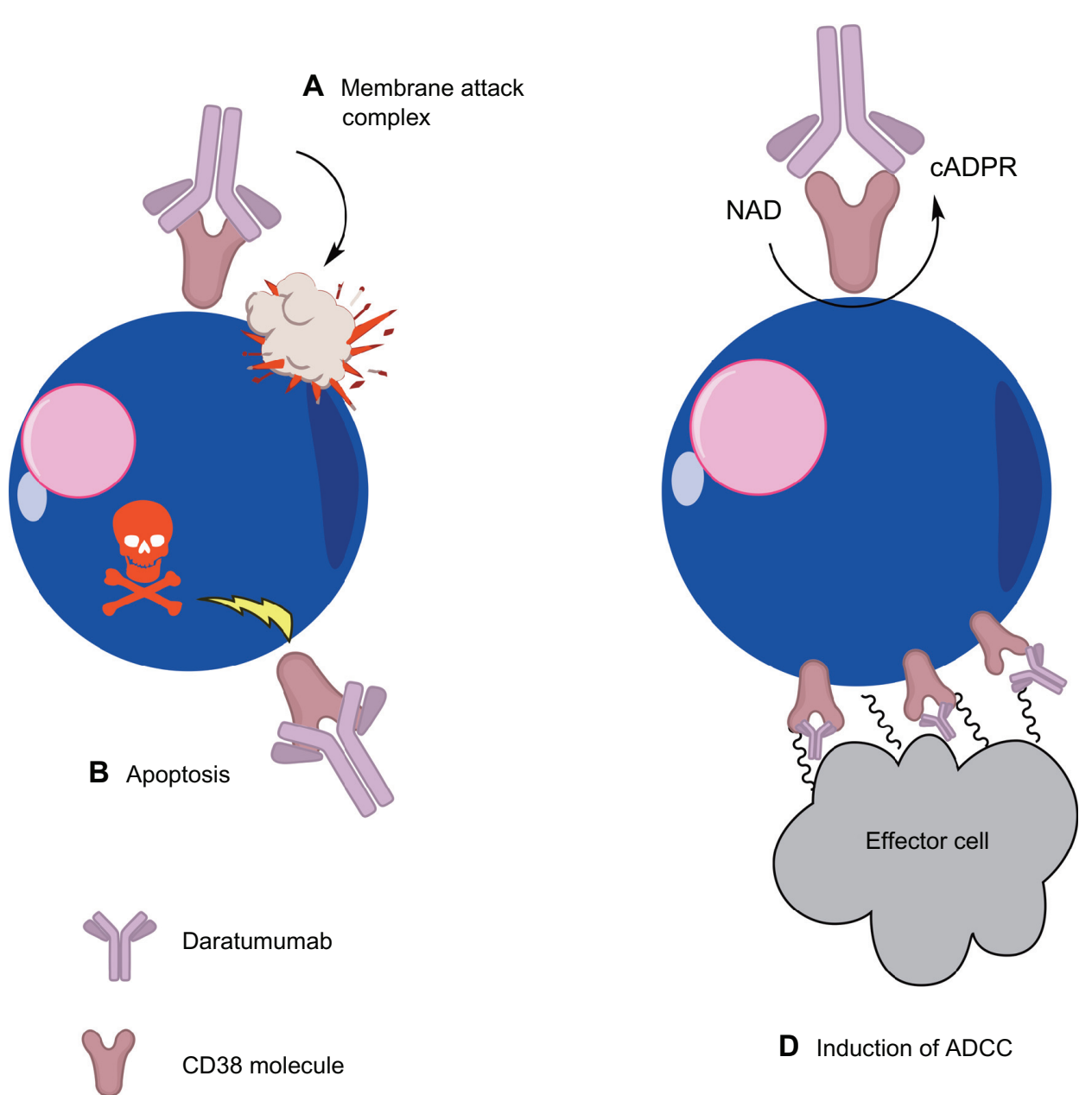

Daratumumab

Modulation of cross-linking of enzymes

Figure 2 Actions of CD38 activation. (A) Induction of CDC; (B) induction of apoptosis; (C) modulation of cross-linking of enzymes; (D) induction of ADCC by engagement with effector cells.

Abbreviations: ADCC, antibody-dependent cellular cytotoxicity; cADPR, cyclic ADPribose; CDC, complement-dependent cytotoxicity; NAD, nicotinamide adenine dinucleotide.

while the remaining had grade 1-2 IREs. Prophylactic administration of steroids managed to reduce the incidence of IREs. Six serious adverse events (SAEs) were assessed as related to daratumumab: one case of grade 3 anemia (dose-limiting toxicity [DLT]) and one case of grade 4 thrombocytopenia, both from the $0.1 \mathrm{mg} / \mathrm{kg}$ cohort; one case of grade 3 elevated aspartate aminotransferase (AST) (DLT) at $1 \mathrm{mg} / \mathrm{kg}$; one patient with grade 2 cytokine release syndrome $(0.1 \mathrm{mg} / \mathrm{kg})$; and two cases of grade 3 and 2, bronchospasm in the $2 \mathrm{mg} / \mathrm{kg}$ and $24 \mathrm{mg} / \mathrm{kg}$ cohorts, respectively. All patients managed to recover from the SAEs after relevant treatments.

With regard to the pharmacokinetic profile of daratumumab, peak plasma levels of the drug were typically reached after the first full dose. Rapid clearance of the drug was observed at low doses, indicating a target-mediated clearance. A high interpatient variability reflects the influence of tumor load on the phar- macokinetics of the drug. The observed plasma concentrations of daratumumab approached predicted values only at higher dose levels, indicating that the impact of target-mediated clearance becomes negligible at higher doses. Overall, the maximal tolerated dose (MTD) has not been reached yet.

Daratumumab displayed a dose-dependent efficacy where greater reduction in paraprotein levels of patients was observed at higher doses and a high area under the curve correlated with prolonged progression-free survival (PFS). Response rates by the International Myeloma Working Group (IMWG) criteria were evaluable for 15 of the 32 (47\%) study patients who received 8 weeks of treatment in doses up to $24 \mathrm{mg} / \mathrm{kg}$ for the update at The American Society of Hematology (ASH) 2012 annual meeting. Four patients (13\%) achieved a partial response, while six patients had a minimal response (19\%). Five patients (16\%) were noted to have 
stable disease. Eight of 12 patients (67\%) receiving $\geq 4 \mathrm{mg} / \mathrm{kg}$ daratumumab had minimal disease or better. Biochemical responses were accompanied by clearance of MM cells from the bone marrow. ${ }^{33}$

Increased daratumumab exposure correlated with longer PFS. ${ }^{33}$

\section{Future directions and potential for combination studies}

Although the Phase I/II study did not assess quality of life issues, it is encouraging to see that, except for IREs, there were no major side effects and the drug was relatively well tolerated..$^{31-33}$ The once-a-week infusion and the uncommon occurrence of severe cytopenias would be seen as favorable, as patients would not need protracted hospital stays as would be expected of any salvage regimens in heavily pretreated patients. The currently planned multicenter Phase II/III studies would shed more light on patients' quality of life with daratumumab treatment. ${ }^{31-33}$

The early results of daratumumab used as a single agent are impressive. In a group of heavily pretreated patients, considerable responses have already been observed with daratumumab monotherapy. ${ }^{31-33}$ Dose-escalation studies ${ }^{31}$ of daratumumab are in progress. When the MTD is established, an extension study with daratumumab as monotherapy will be conducted..$^{31}$ However, as the current clinical practice shows that the future of successful MM treatment lies in the use of drug-combination regimens, it appears essential to identify regimens in which individual components synergize to obtain the greatest achievable effects. Therefore, it comes as no surprise that daratumumab will be explored as a component of multidrug chemotherapy regimens including combinations with bortezomib and lenalidomide. ${ }^{34,35}$ This is predicated on convincing preclinical data showing the potential and synergism of these combinations. The potential role of cytotoxicity induced by the anti-CD38 antibody and the activation of effector cells with the immunomodulatory effects of lenalidomide could make this a very attractive combination for further clinical testing. In addition, with the current focus in MM therapy on consolidating and maintaining responses when a minimal residual disease state is attained, extended exposure for up to 24 months in MM patients with $8 \mathrm{mg} / \mathrm{kg}$ daratumumab as monotherapy will also be evaluated based ${ }^{31,32}$ on the initial observation of a longer PFS with longer exposure and the good safety and tolerability profile of daratumumab.

\section{Conclusion and place in therapy}

Daratumumab is a novel, high-affinity, therapeutic human monoclonal antibody against a unique CD38 epitope with broad-spectrum killing activity. It has shown a favorable safety profile as monotherapy in patients with relapsed/ refractory MM. It also demonstrates significant single agent activity in this group of multiply pretreated patients. Abundant preclinical data ${ }^{28,29}$ supports its use in combination therapy and combination trials with bortezomib and lenalidomide are under way. ${ }^{34,35}$ In spite of recent advances, MM remains an incurable disease, and new approaches that induce long-term tumor regression with little cross-resistance with existing drugs are urgently needed. Immunotherapy with mAbs is a promising area of development that focuses on strategies to elicit myelomaspecific immune responses to eliminate the malignant plasma cells selectively. The introduction of daratumumab as a new class of drug in the battle against MM is timely. The eagerly anticipated results of further clinical studies in the future will hopefully fill the emergent need for improved treatment strategies in MM.

\section{Disclosure}

The authors report no conflicts of interest in this work.

\section{References}

1. SEER Cancer Statistics Review 1975-2008 [webpage on the Internet]. National Cancer Institute; 2008. Available from http://seer.cancer.gov/csr/1975_2008/ results_merged/sect_18_myeloma.pdf. Accessed April 12, 2013.

2. San Miguel JF, Schlag R, Khuageva NK, et al; VISTA Trial Investigators. Bortezomib plus melphalan and prednisone for initial treatment of multiple myeloma. N Engl J Med. 2008;359(9):906-917.

3. Facon T, Mary JY, Hulin C, et al; Intergroupe Francophone du Myélome. Melphalan and prednisone plus thalidomide versus melphalan and prednisone alone or reduced-intensity autologous stem cell transplantation in elderly patients with multiple myeloma (IFM 99-06): a randomised trial. Lancet. 2007;370(9594):1209-1218.

4. Hulin C, Facon T, Rodon P, et al. Efficacy of melphalan and prednisone plus thalidomide in patients older than 75 years with newly diagnosed multiple myeloma: IFM 01/01 trial. J Clin Oncol. 2009;27(22):3664-3670.

5. Wijermans P, Schaafsma M, Termorshuizen F, et al; Dutch-Belgium Cooperative Group HOVON. Phase III study of the value of thalidomide added to melphalan plus prednisone in elderly patients with newly diagnosed multiple myeloma: the HOVON 49 Study. J Clin Oncol. 2010; 28(19):3160-3166.

6. Short KD, Rajkumar SV, Larson D, et al. Incidence of extramedullary disease in patients with multiple myeloma in the era of novel therapy, and the activity of pomalidomide on extramedullary myeloma. Leukemia. 2011;25(6):906-908.

7. Gangatharan SA, Carney DA, Prince HM, et al. Emergence of central nervous system myeloma in the era of novel agents. Hematol Oncol. 2012;30(4):170-174.

8. Reichardt VL, Okada CY, Liso A, et al. Idiotype vaccination using dendritic cells after autologous peripheral blood stem cell transplantation for multiple myeloma - a feasibility study. Blood. 1999;93(7):2411-2419.

9. Reichardt VL, Milazzo C, Brugger W, Einsele H, Kanz L, Brossart P. Idiotype vaccination of multiple myeloma patients using monocytederived dendritic cells. Haematologica. 2003;88(10):1139-1149.

10. Titzer S, Christensen O, Manzke O, et al. Vaccination of multiple myeloma patients with idiotype-pulsed dendritic cells: immunological and clinical aspects. Br J Haematol. 2000;108(4):805-816. 
11. Lim SH, Bailey-Wood R. Idiotypic protein-pulsed dendritic cell vaccination in multiple myeloma. Int J Cancer. 1999;83(2):215-222.

12. Liso A, Stockerl-Goldstein KE, Auffermann-Gretzinger S, et al. Idiotype vaccination using dendritic cells after autologous peripheral blood progenitor cell transplantation for multiple myeloma. Biol Blood Marrow Transplant. 2000;6(6):621-627.

13. Di Bernardo A, Macor P, Guarnotta C, et al. Humoral immunotherapy of multiple myeloma: perspectives and perplexities. Expert Opin Biol Ther. 2010;10(6):863-873.

14. Weiner LM, Surana R, Wang S. Monoclonal antibodies: versatile platforms for cancer immunotherapy. Nat Rev Immunol. 2010;10(5): 317-327.

15. Zonder JA, Mohrbacher AF, Singhal S, et al. A phase 1, multicenter, open-label, dose escalation study of elotuzumab in patients with advanced multiple myeloma. Blood. 2012;120(3):552-559.

16. Lonial S, Vij R, Harousseau JL, et al. Elotuzumab in combination with lenalidomide and low-dose dexamethasone in patients with relapsed/ refractory multiple myeloma: results of a phase 1 study. J Clin Oncol. 2012;30(16):1953-1959.

17. Richardson PG, Moreau P, Jakubowiak AJ, et al. Elotuzumab in combination with lenalidomide and dexamethasone in patients with relapsed multiple myeloma: interim results of a phase 2 study. Blood. (ASH Annual Meeting Abstracts). 2010;116(2):Abstract 986.

18. Deaglio S, Mehta K, Malavasi F. Human CD38: a (r)evolutionary story of enzymes and receptors. Leuk Res. 2001;25(1):1-12.

19. Malavasi F, Funaro A, Roggero S, Horenstein A, Calosso L, Mehta K. Human CD38: a glycoprotein in search of a function. Immunol Today. 1994;15(3):95-97.

20. Lee HC. Structure and enzymatic functions of human CD38. Mol Med. 2006;12(11-12):317-323

21. Chan CS, Wormsley SB, Pierce LE, Peter JB, Schechter GP. B-cell surface phenotypes of proliferating myeloma cells: target antigens for immunotherapy. Am J Hematol. 1990;33(2):101-109.

22. Lin P, Owens R, Tricot G, Wilson CS. Flow cytometric immunophenotypic analysis of 306 cases of multiple myeloma. Am J Clin Pathol. 2004;121(4):482-488.

23. Stevenson FK, Bell AJ, Cusack R, et al. Preliminary studies for an immunotherapeutic approach to the treatment of human myeloma using chimeric anti-CD38 antibody. Blood. 1991;77(5):1071-1079.

24. Ellis JH, Barber KA, Tutt A, et al. Engineered anti-CD38 monoclonal antibodies for immunotherapy of multiple myeloma. J Immunol. 1995; 155(2):925-937.

25. de Weers M, Tai YT, van der Veer MS, et al. Daratumumab, a novel therapeutic human CD38 monoclonal antibody, induces killing of multiple myeloma and other hematological tumors. J Immunol. 2011; 186(3):1840-1848.

26. Overdijk MD, Verploegen S, Marijn B, van Egmond M, Groen RWJ. Phagocytosis is a mechanism of action for daratumumab. Blood (ASH Annual Meeting Abstracts). 2012;120:Abstract 4054.
27. Noort WA, Groen RWJ, Raymakers R, Aalders L, Hofhuis FM, Van Kessel B. Daratumumab, a novel therapeutic human CD38 monoclonal antibody, induces killing of refractory patient-derived multiple myeloma cells, growing in a novel humanized mouse MM model. Blood (ASH Annual Meeting Abstracts). 2012;120:Abstract 940.

28. van der Veer MS, de Weers M, van Kessel B, et al. Towards effective immunotherapy of myeloma: enhanced elimination of myeloma cells by combination of lenalidomide with the human CD38 monoclonal antibody daratumumab. Haematologica. 2011;96(2):284-290.

29. van der Veer MS, de Weers M, van Kessel B, et al. The therapeutic human CD38 antibody daratumumab improves the anti-myeloma effect of newly emerging multi-drug therapies. Blood Cancer J. 2011; 1(10):e41.

30. Gimsing P, Plesner T, Nahi H, et al. A phase I/II, dose-escalation study of daratumumab, a CD38 Mab in patients with multiple myeloma: preliminary safety data. Program and abstracts of the 53rd American Society of Hematology Annual Meeting and Exposition; December 10-13, 2011; San Diego, CA, USA. Abstract 1873.

31. Plesner T, Lokhorst H, Gimsing P, Nahi H, Lisby S, Richardson PG. Daratumumab, a CD38 mab, for the treatment of relapsed/refractory multiple myeloma patients: preliminary efficacy data from a multicenter phase I/II study. Program and abstracts of the 2012 American Society of Clinical Oncology Annual Meeting; June 1-5, 2012; Chicago, IL, USA. Abstract 8019.

32. Lokhorst H, Gimsing P, Nahi H, Richardson P, Plesner T, Lisby S. Daratumumab, a CD38 monoclonal antibody in patients with multiple myeloma: preliminary efficacy and pharmacokinetics data from a dose-escalation phase I/II study. Program and abstracts of the 17th Congress of the European Hematology Association; June 14-17, 2012; Amsterdam, The Netherlands. Abstract 1143.

33. Plesner T, Lokhorst H, Gimsing P, Nahi H, Lisby S, Richardson PG. Daratumumab, a CD38 monoclonal antibody in patients with multiple myeloma: data from a dose-escalation phase I/II study. ASH Annual Meeting Abstracts. 2012;120:73.

34. Genmab. An open-label, international, multicenter, dose escalating phase I/II trial investigating the safety of daratumumab in combination with bortezomib and dexamethasone in patients with relapsed or refractory multiple myeloma. In: ClinicalTrials.gov [website on the Internet]. Bethesda, MD: US National Library of Medicine; 2012 [updated June 20, 2012]. Available from: http://www.clinicaltrials.gov/ct2/show/NCT01620879. NLM identifier: NCT01620879. Accessed June 20, 2012.

35. Genmab. An open label, international, multicenter, dose escalating phase I/II trial investigating the safety of daratumumab in combination with lenalidomide and dexamethasone in patients with relapsed or relapsed and refractory multiple myeloma. In: ClinicalTrials.gov [website on the Internet]. Bethesda, MD: US National Library of Medicine; 2012 [updated March 4, 2013]. Available from: http://www. clinicaltrials.gov/ct2/show/NCT01615029?term=daratumumab\&rank=2. NLM identifier: NCT01615029. Accessed March 4, 2013.
Blood and Lymphatic Cancer: Targets and Therapy

\section{Publish your work in this journal}

Blood and Lymphatic Cancer: Targets and Therapy is an international, peer-reviewed, open access journal focusing on blood and lymphatic cancer research, identification of therapeutic targets and the optimal use of preventative and integrated treatment interventions to achieve improved outcomes, enhanced survival and quality of life for the

\section{Dovepress}

cancer patient. The manuscript management system is completely online and includes a very quick and fair peer-review system. Visit http://www.dovepress.com/testimonials.php to read real quotes from published authors. 